\section{MUC1 marks collecting tubules, renal vesicles, comma- and S-shaped bodies in human developing kidney}

\author{
D. Fanni, ${ }^{1}$ N. Iacovidou, ${ }^{2}$ A. Locci, ${ }^{1}$ \\ C. Gerosa, ${ }^{1}$ S. Nemolato, ${ }^{1}$ P. Van Eyken, ${ }^{3}$ \\ G. Monga, ${ }^{4}$ S. Mellou, ${ }^{5}$ G. Faa, ${ }^{1}$ V. Fanos ${ }^{6}$ \\ 'Department of Pathology, University \\ of Cagliari, Italy \\ 2Second Department of Obstetrics and \\ Gynecology, National and Kapodistrian \\ University of Athens, Medical School, \\ Aretaieio Hospital, Athens, Greece \\ ${ }^{3}$ Department of Pathology, K.U. Leuven, \\ Belgium \\ ${ }^{4}$ Department of Pathology, University \\ of Piemonte Orientale, Novara, Italy; \\ ${ }^{5}$ Department of Pathology, Aretaieio \\ Hospital, Athens, Greece \\ ${ }^{6}$ Department of Pediatrics and Clinical \\ Medicine, University of Cagliari, Italy
}

\section{Abstract}

MUC1 is a transmembrane glycoprotein, apically expressed in most epithelial cells, used in the differential diagnosis of carcinomas and for discrimination of tumors of non-epithelial origin showing epithelioid features. Little attention has been paid so far though, on its possible significance in embryonic tissues. A preliminary study from our group revealed MUC1 expression in the cap mesenchymal cells during human nephrogenesis, suggesting a role for MUC1 in the process of mesenchymal-toepithelial transition. This study aimed at investigating the expression pattern of MUC1 in various developing structures of human fetal kidney. Expression of MUCl was examined in kidneys of 5 human fetuses. MUC1 immunoreactivity was detected in ureteric bud tips, in collecting tubules, in cap mesenchymal cells undergoing the initial phases of mesenchymalto-epithelial transition, in renal vesicles, comma-bodies, and S-shaped bodies. Our previous preliminary report suggested a role for MUCl in the initial phases of the process of mesenchymal-to-epithelial transition. The present data suggest that MUCl expression characterizes multiple structures during human nephrogenesis, from the ureteric bud, to the initial phases of mesenchymal-to-epithelial transition and that MUC1 should be added to the genes activated during the process of mesenchymal-to-epithelial transition in the cap mesenchyme of human kidney.

\section{Introduction}

Human mucins may be classified i) in extracellular mucins, involved mainly in coating and protecting epithelial surfaces of gastrointestinal and respiratory tract and ii in membrane-tethered mucins. ${ }^{1}$ Human membrane-bound mucins are designated as MUC, whereas mucins of other species are designated as Muc. ${ }^{2}$ Several transmembrane mucins have been identified so far. $^{3}$ Structurally, transmembrane mucins consist of a large extracellular domain, mainly composed of a variable number of the tandemrepeat (TR) domains, in addition of a single membrane-spanning domain and of a cytoplasmic tail. ${ }^{4}$

Previously known as epithelial membrane antigen (EMA), ${ }^{5}$ MUC1 is a high molecular weight transmembrane glycoprotein, which is apically expressed in most epithelial cells of human tissues. ${ }^{6}$ Most studies on MUC1/EMA expresssion have dealt with tumor pathology, in an attempt to identify its possible role as a marker in the differential diagnosis of carcinomas, including that of prostate, ${ }^{7}$ breast, ${ }^{8}$ and kidney ${ }^{9}$ and for the discrimination of tumors of non-epithelial origin exhibiting epithelioid features. ${ }^{10}$ Little attention, though, has been paid on its possible role in normal and embryonic tissues. ${ }^{5,11}$ In a recent study on MUC1 expression during rat embryonic development, kidney was among the most reactive tissues, both in foetuses and in neonatal rats. ${ }^{12}$ On the contrary, a study on MUC1 expression in normal feline tissues did not show any significant expression in renal cells. ${ }^{13}$ High level expression of MUC1 during kidney development was first reported by Chambers et al., apparently restricted to the collecting ducts. ${ }^{14}$ Further studies on human mucin genes during renal development, revealed expression of MUCl also in the metanephric blastema and in the ureteric buds at 9.5 weeks of gestation, while at 6 months postnatally, MUC1 was restricted to distal tubules and collecting ducts. ${ }^{15}$ A recent preliminary study by our group ${ }^{16}$ revealed MUC1 expression in the cap mesenchymal cells during human nephrogenesis, suggesting a role for MUC1 in the process of mesenchymal-to-epithelial transition that characterizes stem/progenitor renal cells and gives rise to nephrons.

Taking in consideration this data, the present study aimed at investigating the expression pattern of MUCl in various renal structures of the developing human fetal kidney in utero, at different gestational ages.
Correspondence: Daniela Fanni, Department of Pathology, S.Giovanni di Dio Hospital, University of Cagliari, via 0spedale 54, 09124 Cagliari, Italy. Tel. +39.070.6092372 - Fax: +39.070 .6092370 .

E-mail: fandan73@yahoo.it

Key words: MUCl, immunohistochemistry, fetal kdney, nephrogenesis, renal vesicles, comma and S-shaped bodies, collecting tubules.

Contributions: DF, conception, design, data interpretation; NI, PVE, GM, preparation and manuscript critical revision; AL, SN, design and analysis of data; CG, design and interpretation of data; SM, manuscript preparation; GF, VF, critical revision of the manuscript.

Conflict of interests: the authors declare no conflict of interests.

Received for publication: 18 February 2012. Accepted for publication: 14 May 2012

This work is licensed under a Creative Commons Attribution NonCommercial 3.0 License (CC BYNC 3.0).

(C) Copyright D. Fanni et al., 2012

Licensee PAGEPress, Italy

European Journal of Histochemistry 2012; 56:e40 doi:10.4081/ejh.2012.e40

\section{Materials and Methods}

Five human foetuses with gestational age ranging from 11-26 weeks were included in the present study. All procedures performed were in accordance with the ethical national standards of the responsible committee on human experimentation. The clinical data is depicted in Table 1. At macroscopic examination, no congenital malformations were observed. Kidneys were formalin-fixed and routinely processed. Immunohistochemistry for MUC1 was performed using the labelled streptavidin-biotin complex system (LSAB2, Dako) in a Dako autostainer (DakoCytomation, Carpintera, CA, USA). Heat-induced antigen retrieval was carried out by steaming unstained sections in Target Retrieval Solution (Dako Denmark A/S, Glostrup, Denmark) for 30 min. Mouse antiMUC1 monoclonal antibody in 1:50 dilution was incubated for $20 \mathrm{~min}$ at room temperature. Sections were processed with streptavidinbiotin complex system (LSAB2, Dako) in a Dako autostainer (DakoCytomation) and Target Retrieval Solution (Dako Denmark A/S). Negative controls samples were incubated without mouse anti-MUC1 monoclonal antibody. Sections were counterstained with Mayer's hematoxylin. 


\section{Results}

MUC1 immunoreactivity was detected in all 10 fetal kidneys examined and no significant difference were observed between the right and the left kidney, in any of the cases examined. MUC1 immunostaining was present in all renal regions, extending from the hylum towards the cortex. Glomerular structures were specifically devoid of MUC1 at all stages of their development. No significant reactivity for MUC1 was detected in mature proximal and distal tubules, as well as in Henle tubules. The following compartments were characterized by MUC1 expression: i) ureteric bud tips and collecting tubules originating from them; ii) cap mesenchymal cells undergoing the initial phases of mesenchymal epithelial transition and iii) renal vesicles, comma-bodies, and Sshaped bodies.

Ureteric buds branching into the metanephric mesenchyme were shown to be strongly positive for MUC1 expression examined at all gestational ages in this study. MUC1 Immunoreactivity was observed mainly in their lumen and at the apical border of the epithelial cells (Figure 1). The vast majority of renal vesicles were also positive for MUC1 expression at all gestational ages, with the immunostaining located inside the vesicle lumen and in the cytoplasm of newly formed epithelial cells (Figure 2). Comma- and Sshaped bodies were consistently MUC1 positive (Figure $3 \mathrm{a}, \mathrm{b})$. In addition, MUC1-expressing cells were detected in a subset of cap mesenchymal aggregates, as previously described. ${ }^{14}$

Glomerular structures were specifically
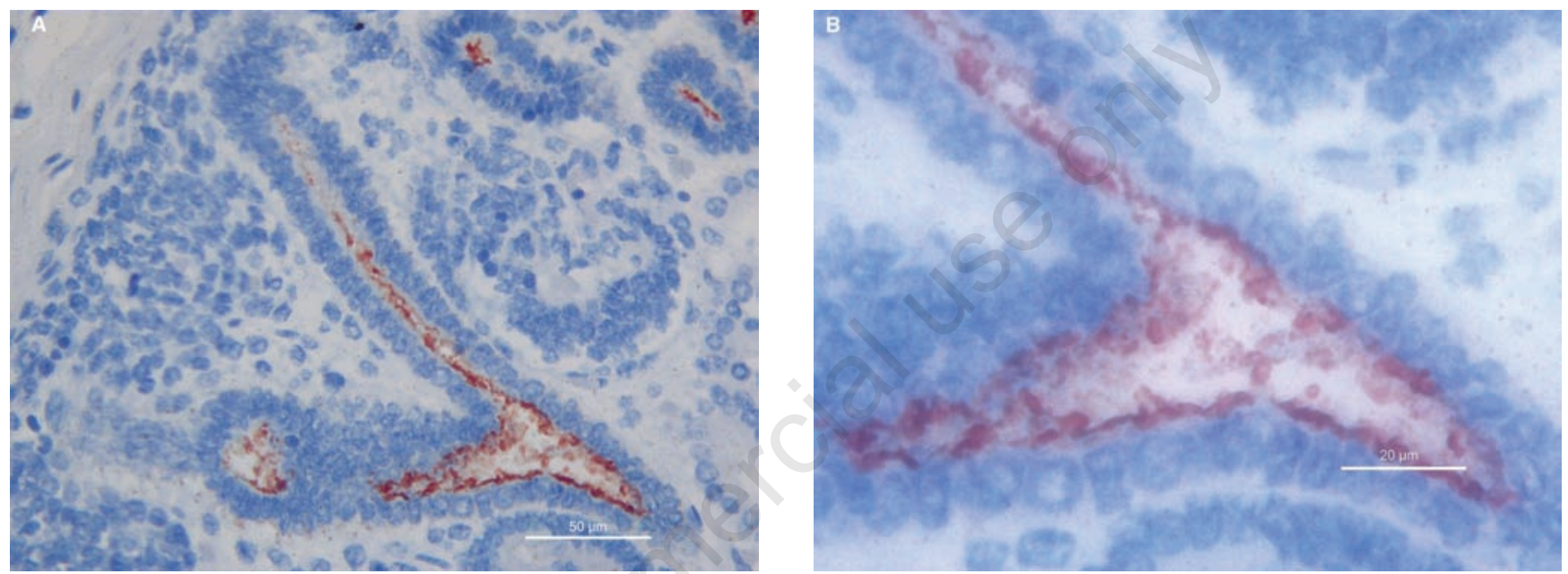

Figure 1. MUC1 immunoreactivity in the lumen and at the apical border of the epithelial cells in ureteric bud. A) $40 \mathrm{hpf}$, scale bar: 50 $\mu \mathrm{m}$; B) $100 \mathrm{hpf}$, scale bar: $20 \mu \mathrm{m}$.

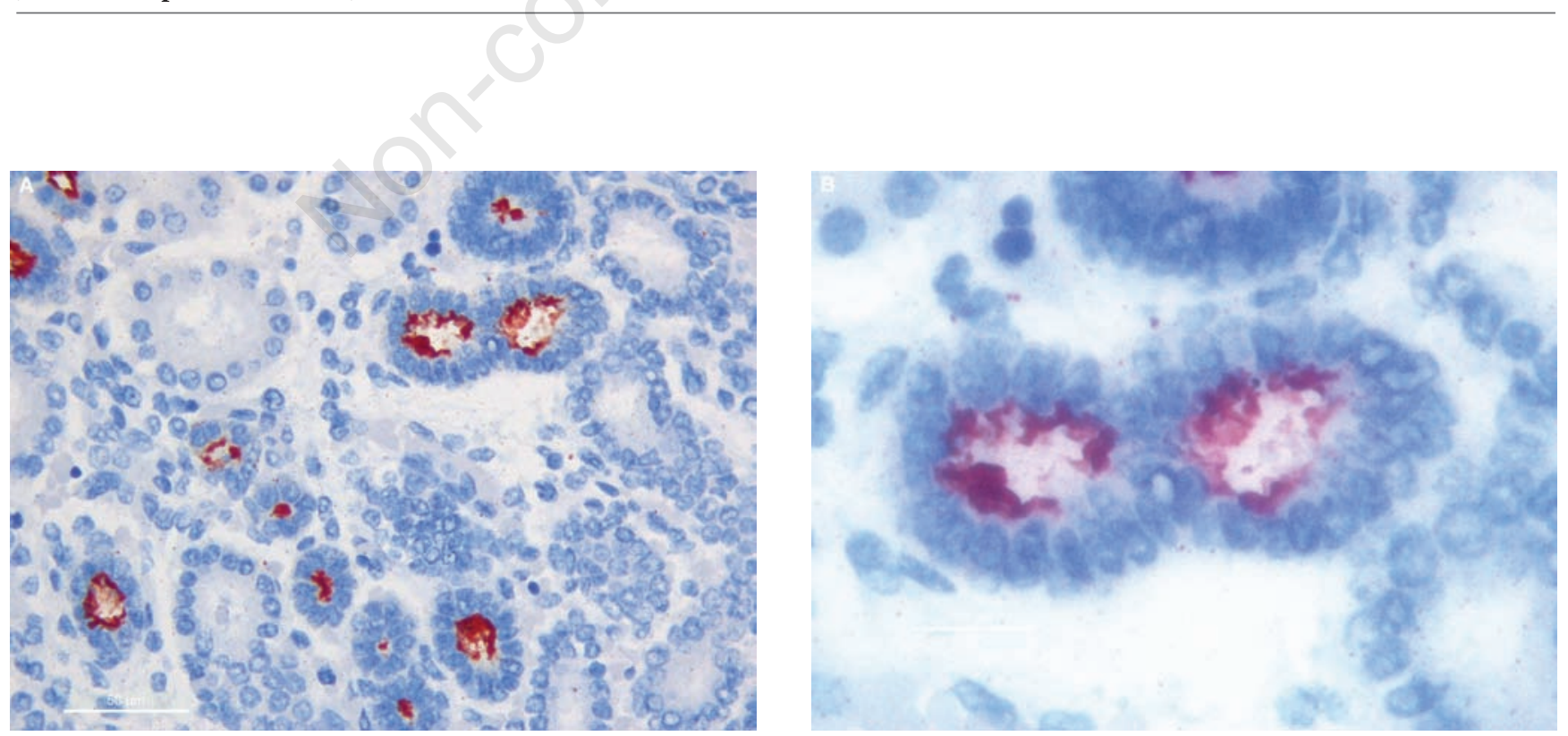

Figure 2. In renal vesicles MUC1 expression is located inside the vesicle lumen and in the cytoplasm of newly formed epithelial cells. A) $40 \mathrm{hpf}$, scale bar: $50 \mu \mathrm{m}$; B) $100 \mathrm{hpf}$, scale bar: $20 \mu \mathrm{m}$. 
devoid of MUC1, at all stages of their development. No significant MUC1 reactivity was detected in mature proximal and distal tubules, as well as in Henle tubules (Figure 4)

No significant alteration was found for MUC1 expression during gestation: MUC1 immunoreactivity appeared well compartimentalized examined at all gestational ages, with mild differences in the degree of reactivity.

\section{Discussion}

Human kidney development, given its complexity, has become a paradigm for the fascinating phenomenon of branching morphogen- esis, for the transformation of mesenchymal cells into epithelial structures, the so called mesenchymal-to-epithelial transition, and for human organogenesis. ${ }^{17}$ This complex process which is still not clearly elucidated, requires complex interactions between epithelial and mesenchymal cells, the former originating from the ureteric bud and the latter from the metanephric mesenchyme, eventually leading to the coordinated development of multiple highly specialized cell types, a peculiar feature of the renal architectural and functional complexity. ${ }^{18}$ The complex process of mesenchymal-to-epithelial cells transition occurs in the cap mesenchymal cells, the self-renewing progenitor cells located around the branching ureteric bud tips. ${ }^{19,20}$ Epithelial differentiation involves transition from a comparatively disor- dered mesenchyme to an organized simple epithelium. Morphological changes are paralleled by the activation of a large number of epithelial genes. ${ }^{19}$

The application of new techniques, such as immunocytochemistry and molecular biology, to the study of cell types involved in renal morphogenesis has been a key challenge, leading to better understanding of kidney development during pre- and post-natal life. ${ }^{20,21} \mathrm{~A}$ recent preliminary study by our group on MUC1 expression in the foetal human kidney first evidenced MUC1 expression in a subset of cap mesenchymal cells aggregates, suggesting a role for this protein in the initial phases of the process of mesenchymal-to-epithelial transition. ${ }^{16}$ In the present study we report that MUC1 expression is not restricted to the cap
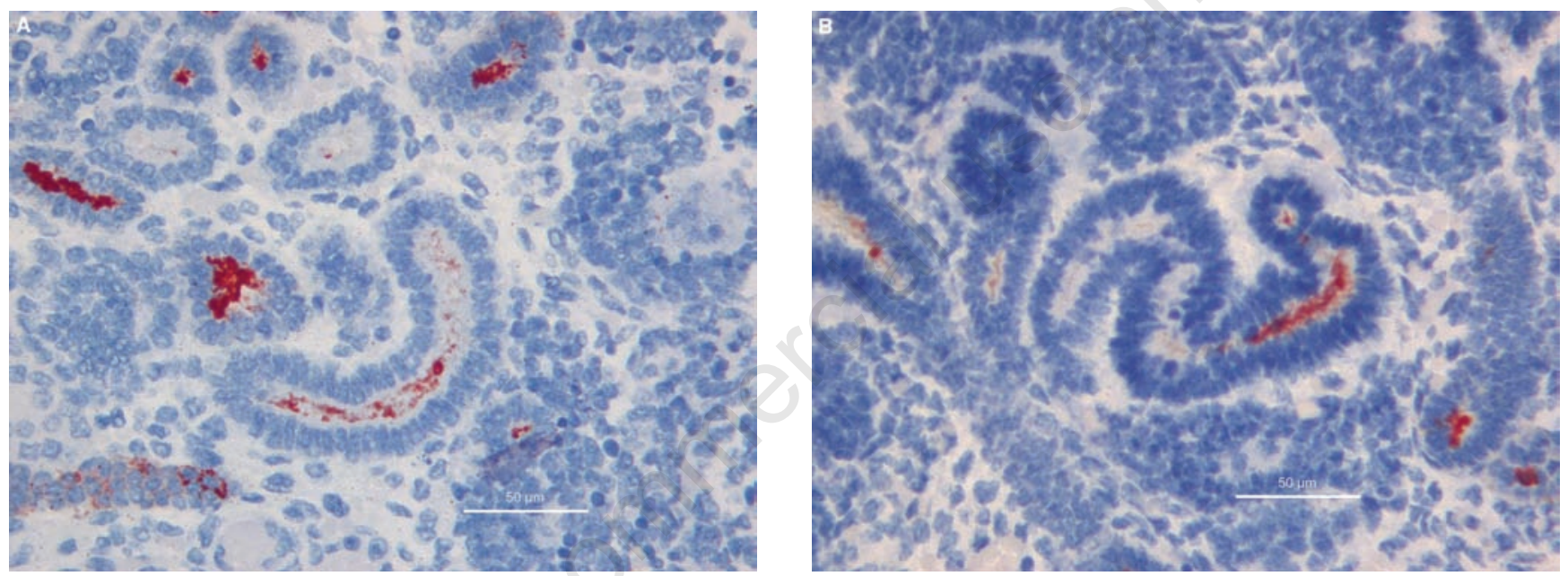

Figure 3. A) Comma-shaped body reactivity; $40 \mathrm{hpf}$, scale bar: $50 \mu \mathrm{m}$. B). S-Shaped bodies; $40 \mathrm{hpf}$, scale bar: $50 \mu \mathrm{m}$.

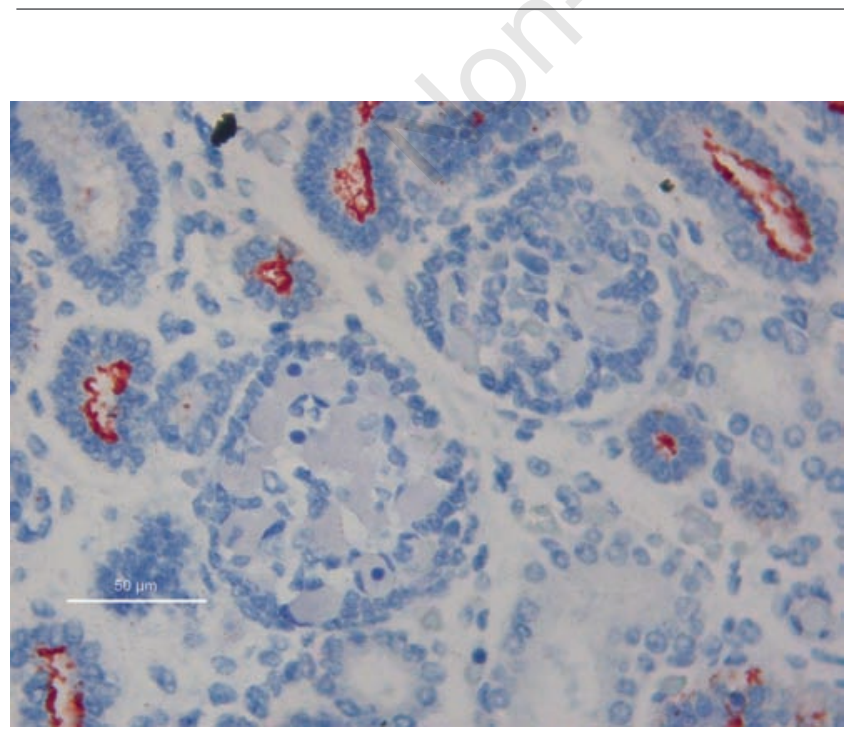

Figure 4. Absence of MUC1 immunoreactivity in glomeruli; $40 \mathrm{hpf}$, scale bar: $50 \mu \mathrm{m}$. 
mesenchyme, but it characterizes multiple steps during human nephrogenesis.

An interesting datum from the present study is the contemporary detection of MUC1 in the renal structures originating from the ureteric bud and in those emerging from the metanephric mesenchyme. Previous immunohistochemical studies carried out in the developing human kidney, were characterized by protein expression patterns restricted to either of these compartments and often to one single renal structure. Thymosin-beta-10 was found to be expressed in proximal and distal tubules, ${ }^{20} \mathrm{CD} 10$ was detected only in the proximal tubules, ${ }^{21}$ whereas Wt1 was found to be mainly associated with the glomeruli, playing a role in the development and maturation of podocytes. ${ }^{22}$ MUC1 represents, to the best of our knowledge, the first protein marking both the collecting tubules, originating from the ureteric bud and the epithelial structures originating from the cap mesenchyme, including renal vesicles, comma- and S-shaped bodies.

In fact, MUC1 was strongly expressed in the S-shaped bodies, including the proximal segments that are known to generate glomeruli. This finding is a new information to be added to the complex picture of gene products involved in human nephrogenesis.

No significant difference was observed in MUC1 expression in kidneys from foetuses of different gestational age. This finding indicates that MUC1 expression is not associated to the duration of gestation, but to the degree of development of renal structures. Since nephrogenesis is an on-going process in humans continuing in the perinatal period, we could hypothesize a continuous MUC1 expression in kidney until then, and a down-regulation when active nephrogenesis is completed.

In conclusion, this data clearly suggests that MUC1 plays a role during human nephrogenesis. MUC1 could serve as a marker of structures originating from the ureteric bud and should be added to the large number of epithelial genes marking the initial phases of mesenchymal-to-epithelial transition occurring in cap mesenchymal cells. Further studies are needed in order to gain better understanding of the associations between the expression patterns of these genes.

\section{Reference}

1. Mall AS. Analysis of mucins: role in laboratory diagnosis. J Clinl Pathol 2008;61:101824.

2. Chaturvedi P, Singh AP, Batra SK. Structure, evolution, and biology of the MUC4 mucin. FASEB J 2008;22:966-81.

3. Bafna S, Kaur S, Batra SK. Membranebound mucins: the mechanistic basis for alterations in the growth and survival of cancer cells. Oncogene 2010;29:2893-904.

4. Moniaux N, Escande F, Porchet N, Aubert JP, Batra SK. Structural organization and classification of the human mucin genes. Front Biosci 2001;6:D1192-206.

5. Sloane JP, Ormerod MG. Distribution of epithelial membrane antigen in normal and neoplastic tissues and it value in diagnostic tumor pathology. Cancer 1981;47:1786-95.

6. Patton S, Gendler SJ, Spicer AP. The epithelial mucin, MUC1, of milk, mammary gland and other tissues. Biochim Biophys Acta1995;1241:407-23.

7. Singh AP, Chauhan SC, Bafna S, Johansson SL, Smith LM, Moniaux N, et al. Aberrant expression of transmembrane mucins, MUC1 and MUC4, in human prostate carcinomas. Prostate 2006; 66:421-9.

8. Hattrup CL, Gendler SJ. MUC1 alters oncogenic events and transcription in human breast cancer cells. Breast Cancer Res 2006;8:R37.

9. Langner C, Ratschek M, Rehak P, Schips L, Zigeuner R. Expression of MUC1 (EMA) and E-cadherin in renal cell carcinoma: a systematic immunohistochemical analysis of 188 cases. Modern Pathol 2004;17:180-8.

10. Kawano T, Ahmad R, Nogi H, Agata N, Anderson K, Kufe D. MUC1 oncoprotein promotes growth and survival of human multiple myeloma cells. Int $\mathrm{J}$ Oncol 2008;33:153-9.

11. Corfield AP, Carroll D, Myerscough N, Probert CS. Mucins in the gastrointestinal tract in health and disease. Front Biosci 2001;6:D1321-57.

12. Lacunza E, Ferretti V, Barbeito C, Segal-
Eiras A, Croce MV. Immunohistochemical evidence of Mucl expression during rat embryonic development. Eur J Histochem 2010;54:e49.

13. Lacunza E, Abba MC, Segal-Eiras A, Croce MV. Identification and expression of the epithelial Muc1 mucin in normal feline tissues. Vet Immunol Immunop. 2009;130):17-24.

14. Chambers JA, Hollingsworth MA, Trezise $\mathrm{AE}$, Harris A. Developmental expression of mucin genes MUC1 and MUC2. J Cell Sci 1994;107:413-24.

15. Leroy X, Devisme L, Buisine MP, Copin MC, Aubert S, Gosselin B, et al. Expression of human mucin genes during normal and abnormal renal development. Am J Clin Pathol 2003;120:544-50.

16. Fanni D, Fanos V, Monga G, Gerosa C, Nemolato S, Locci A, et al. MUC1 in mesenchymal-to-epithelial transition during human nephrogenesis: changing the fate of renal progenitor/stem cells? J Matern Fetal Neonatal Med 2011;24(Suppl.2):63-6.

17. Dressler GR. Epigenetics, development, and the kidney. J Am Soc Nephrol 2008;19: 2060-7.

18. Dressler GR. The cellular basis of kidney development. Annu Rev Cell Dev Biol 2006;22:509-29.

19. Pleniceanu 0, Harari-Steinberg 0, Dekel B. Concise review: kidney stem/progenitor cells: differentiate, sort out, or reprogram? Stem Cells 2010;28:1649-60.

20. Gerosa C, Fanni D, Nemolato S, Locci A, Marinelli V, Cabras T, et al. Thymosin beta10 expression in developing human kidney. J Matern Fetal Neonatal Med 2010;23(Suppl.3):125-8.

21. Faa G, Gerosa C, Fanni D, Nemolato S, Marinelli V, Locci A, et al. CD10 in the developing human kidney: immunoreactivity and possible role in renal embryogenesis. J Matern Fetal Neonatal Med 2012;25:904-11.

22. Fanni D, Fanos V, Monga G, Gerosa C, Locci A, Nemolato S, et al. Expression of WT1 during normal human kidney development. J Matern Fetal Neonatal Med 2011;24(Suppl.2):44-7. 